\title{
Percutaneous Endoscopic Gastrostomy in a Patient With Continuous Intrathecal Baclofen Infusion Therapy
}

\author{
Ezekiel Wong Toh Yoon ${ }^{\mathrm{a}, \mathrm{c}}$, Masuki Kobayashi ${ }^{\mathrm{b}}$
}

\begin{abstract}
Percutaneous endoscopic gastrostomy (PEG) is the method of choice in patients requiring long-term enteral tube feeding. In patients with intrathecal baclofen infusion therapy (IBT) pump implantation, infection via the skin and soft tissue may be an issue of concern. The introducer technique for gastrostomy tube insertion may be useful in reducing the risk of peristomal infection. Although the presence of a PEG tube has been reported as a risk factor for implantation site infection (for newly inserted IBT pumps), whether existing IBT pumps are at risk for infection during or after the placement of a PEG tube is not clear. We report a case where PEG was successfully performed using the introducer technique on a patient with an IBT pump implant.
\end{abstract}

Keywords: Percutaneous endoscopic gastrostomy; Enteral nutrition; Spinal cord injury; Muscle spasm; Intrathecal baclofen infusion therapy

\section{Introduction}

Continuous intrathecal baclofen infusion therapy (IBT) makes use of an implanted programmable pump to deliver baclofen, a derivative of gamma-aminobutyric acid (GABA), directly to the cerebral spinal fluid. IBT has been demonstrated to be effective in controlling spasticity and muscle spasms following spinal cord injury $[1,2]$. Patients with spinal cord injury may also suffer from dysphagia or insufficient oral intake, requiring long-term enteral tube nutrition in some cases. Percutaneous endoscopic gastrostomy (PEG) has been established as a minimally invasive procedure to achieve percutaneous tube feeding for long-term enteral nutrition [3, 4]. Although the presence of a PEG tube has been reported as the greatest significant predictor of infection in patients undergoing IBT pump implantation, there is limited literature regarding the safety or rate of infec-

Manuscript accepted for publication February 01, 2017

aDepartment of Internal Medicine (Gastroenterology), Hiroshima Kyoritsu Hospital, Hiroshima City, Japan

bepartment of Neurosurgery, Hibino Hospital, Hiroshima City, Japan

${ }^{\mathrm{c} C o r r e s p o n d i n g ~ A u t h o r: ~ E z e k i e l ~ T o h ~ Y o o n ~ W o n g, ~ D e p a r t m e n t ~ o f ~ I n t e r n a l ~}$ Medicine, Hiroshima Kyoritsu Hospital, 2-20-20 Nakasu Asaminami-ku, Hiroshima City, Japan. Email: easybs@hotmail.com

doi: https://doi.org/10.14740/gr786w tion among patients with IBT undergoing PEG [5]. We herein report the successful PEG tube placement in a 68-year-old man with a previously implanted IBT pump.

\section{Case Report}

A 68-year-old man was referred to our hospital to undergo PEG for long-term enteral tube feeding. About one and a half years ago, he encountered a traffic accident which gave him a cerebral contusion and diffuse axonal injury. He was left with quadriplegia and dysphagia, being tube-fed via a nasogastric tube since the accident. The patient experienced severe muscle spasms from his spinal cord injury and about a year ago had an IBT pump implanted subcutaneously into his left inguinal area to help alleviate his symptoms.

Although muscle and articular contracture was rather severe, physical examination was otherwise unremarkable. Laboratory data were mostly within the normal range and abdominal CT evaluation indicated that PEG was possible. The patient underwent PEG using the introducer technique with a 20 Fr size catheter kit (Intolief PEG kit by Create Medic Co., Ltd, Yokohama, Japan). After determining the puncture site, local anesthesia was applied and gastropexy was performed using a gastropexy device (Fig. 1a). Gastropexy procedure was repeated at least three times surrounding the intended puncture site in a triangular pattern before the puncture needle/trocar was inserted (Fig. 1b). The puncture needle was then removed (Fig. 1c) and a $20 \mathrm{Fr}$ size gastrostomy tube was inserted into the gastric lumen (Fig. 1d). No problems were encountered during the procedure.

Postoperative clinical course was also uneventful and there was no peristomal infection at 1 week after the procedure. After achieving stable intermittent gastric feeding via the PEG tube, the patient was discharged 10 days after the procedure without further complications. Follow-up on day 30 after the procedure confirmed that there were no adverse events related to the IBT pump implant. An abdominal radiograph taken on day 2 after the procedure showed the position of the gastrostomy tube (Fig. 2, yellow arrow) relative to the implanted IBT pump (Fig. 2, red arrow).

\section{Discussion}

Our case demonstrated that PEG is a procedure that can be safely performed in a patient with a previous IBT pump im- 


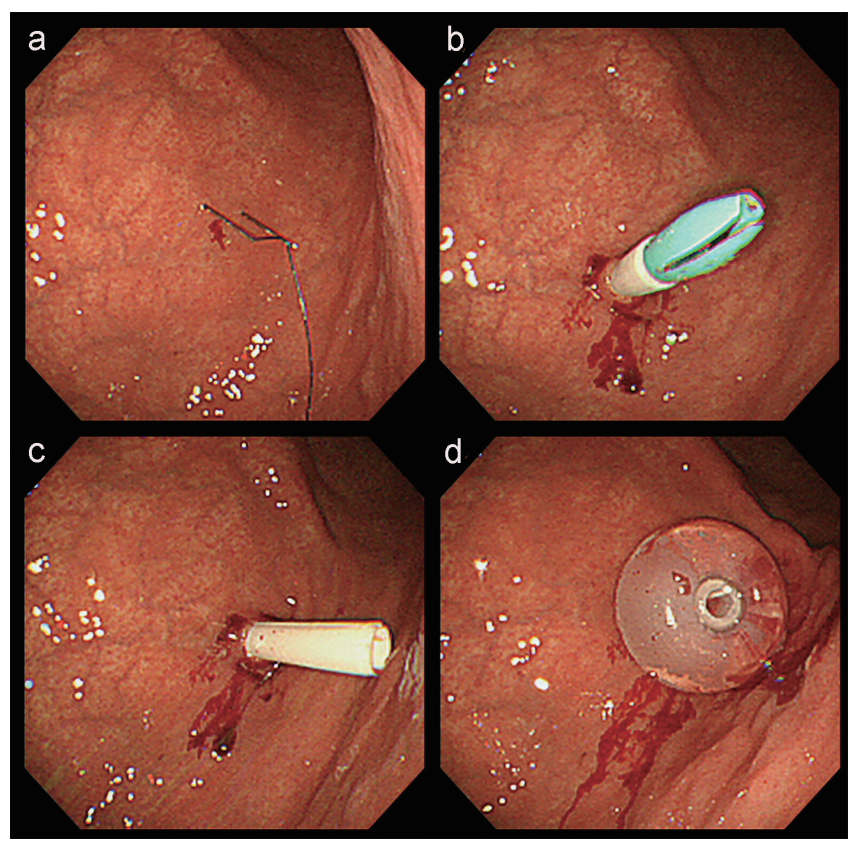

Figure 1. Endoscopic imaging of PEG using the introducer technique in this patient. (a) After determining the puncture site, local anesthesia was applied and gastropexy was performed using a gastropexy device. (b) Gastropexy procedure was repeated at least three times surrounding the intended puncture site in a triangular pattern before the puncture needle/trocar was inserted. (c) The puncture needle was then removed. (d) A $20 \mathrm{Fr}$ size gastrostomy tube was inserted into the gastric lumen.

plantation. Although previous PEG tube placement seems to contribute to IBT pump-related infections, the route of infection remains unclear. However, it should be noted that the most common causative agent in IBT pump-related infection (which may occur in up to $9 \%$ of implanted patients) has been shown to be Staphylococcus aureus [5, 6], which is common in skin and soft tissue infections [7]. Since peristomal infection has also been reported as the most common adverse event after PEG, it is quite plausible that IBT pump-related infection after PEG tube placement occurs mainly through the skin or connective tissue [8]. The occurrence of implantation site infection is not something that can be taken lightly because it may develop to meningitis if not managed properly [9].

Although systemic antimicrobial prophylaxis has been shown to reduce the incidence of post-PEG peristomal infection, it does not totally eliminate the risk of infection [10]. PEG using the introducer technique, in which the gastrostomy tube is inserted without passing through the oral cavity, has also been associated with lower peristomal infection rates compared to conventional pull/push techniques [11]. Nevertheless, this technique is not widely used because the size of the PEG catheter employed is often small. Recently, large-caliber tubes using this technique, such as in our case, can also be safely inserted without increasing the risk of peristomal infection [12]. In our patient, the introducer technique was used along with systemic antimicrobial prophylaxis.

In conclusion, PEG performed after IBT implantation seems to be reasonably safe although there are no large stud-

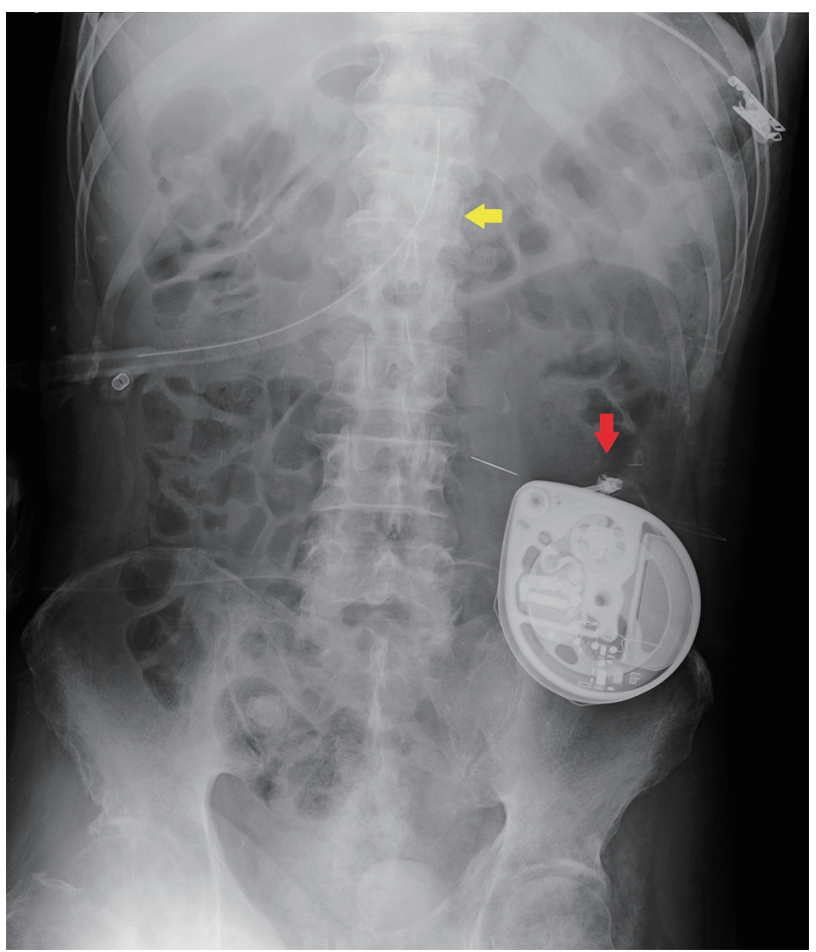

Figure 2. Abdominal radiograph revealing the position of the PEG catheter (yellow arrow) relative to the implanted IBT pump (red arrow).

ies we are aware of to confirm this. If infection does occur mainly via the skin and soft tissue, further measures to avoid IBT pump-related infection after PEG tube placement such as the choice of tube insertion technique as well as systematic antibiotics administration should be strongly considered.

\section{Conflicts of Interest}

None.

\section{References}

1. Lewis KS, Mueller WM. Intrathecal baclofen for severe spasticity secondary to spinal cord injury. Ann Pharmacother. 1993;27(6):767-774.

2. McIntyre A, Mays R, Mehta S, Janzen S, Townson A, Hsieh J, Wolfe D, et al. Examining the effectiveness of intrathecal baclofen on spasticity in individuals with chronic spinal cord injury: a systematic review. J Spinal Cord Med. 2014;37(1):11-18.

3. Gauderer MW, Ponsky JL, Izant RJ, Jr. Gastrostomy without laparotomy: a percutaneous endoscopic technique. J Pediatr Surg. 1980;15(6):872-875.

4. DeLegge MH. Percutaneous endoscopic gastrostomy. Am J Gastroenterol. 2007;102(12):2620-2623.

5. Fjelstad AB, Hommelstad J, Sorteberg A. Infections related to intrathecal baclofen therapy in children and adults: frequency and risk factors. J Neurosurg Pediatr. 
2009;4(5):487-493.

6. Malheiro L, Gomes A, Barbosa P, Santos L, Sarmento A. Infectious Complications of Intrathecal Drug Administration Systems for Spasticity and Chronic Pain: 145 Patients From a Tertiary Care Center. Neuromodulation. 2015;18(5):421-427.

7. Creech CB, Al-Zubeidi DN, Fritz SA. Prevention of Recurrent Staphylococcal Skin Infections. Infect Dis Clin North Am. 2015;29(3):429-464.

8. Richter-Schrag HJ, Richter S, Ruthmann O, Olschewski M, Hopt UT, Fischer A. Risk factors and complications following percutaneous endoscopic gastrostomy: a case series of 1041 patients. Can J Gastroenterol. 2011;25(4):201-206.
9. Bennett MI, Tai YM, Symonds JM. Staphylococcal meningitis following Synchromed intrathecal pump implant: a case report. Pain. 1994;56(2):243-244.

10. Lipp A, Lusardi G. Systemic antimicrobial prophylaxis for percutaneous endoscopic gastrostomy. Cochrane Database Syst Rev. 2013;(11):CD005571.

11. Campoli PM, de Paula AA, Alves LG, Turchi MD. Effect of the introducer technique compared with the pull technique on the peristomal infection rate in PEG: a metaanalysis. Gastrointest Endosc. 2012;75(5):988-996.

12. Toh Yoon EW, Yoneda K, Nakamura S, Nishihara K. Percutaneous endoscopic gastrostomy (PEG) using a novel large-caliber introducer technique kit: a retrospective analysis. Endosc Int Open. 2016;4(9):E990-E996. 